\title{
Artificial Organs and Related Bioinorganic Materials
}

\author{
Prof. Barry Wiling \\ Professor, Department of Computer Science, \\ U.S.M.N Oman \\ barrywiling@usmn.edu
}

\section{Abstract}

Bioinorganic compounds or materials play the momentous role in all living organisms. Artificial organs are generally defined as any device, machine or complex biological structure which is partially or completely synthetic in nature and that could be implanted or integrated into human body to perform the tasks of a particular biological structure which has been damaged and should be replaced due to some medical reasons. Various artificial organs like bone, heart, kidney, liver, lung, pancreas, skin, urinary bladder, auditory brainstem implant, bionic contact lens, cochlear implant, direct acoustic cochlear implant, retinal implant and visual prosthetic parts have been developed. In this paper we are disusing about artificial biomaterial and organs.

Keywords: artificial heart, skin, sensor etc.

\section{INTRODUCTION}

Predominantly these compounds are responsible for the vital activities of an organism such as enzymatic actions, respiration, photosynthesis, metal ion transport etc. In recent time numerous bioinorganic materials have been synthesized partially or completely in the laboratories. These synthetic bioinorganic materials serve as medical boon for mankind as they are the key for development of artificial tissues and organs.

\section{ARTIFICIAL ORGANS}

Artificial Heart:- it is basically a device which provides circulatory support to the patients, those who are waiting for donor hearts or those who need temporary support while their damaged hearts recover. Jarvik-7 was the first successfully implanted artificial heart in human. Ventricular assist device and cardiopulmonary bypass machine are also implanted in human 
bodies to support the failing heart. POLVAD, Phoenix-7, Abiomed AbioCor, SynCardia, MagScrew, Abiomed AbioCor II, Carmat bioprosthetic heart, Frazier-Cohn are the different prototypes of the artificial heart. These devices are generally made up of metal alloys, flexible silicones, chemically treated animal tissues, bio materials, electronic sensors etc.

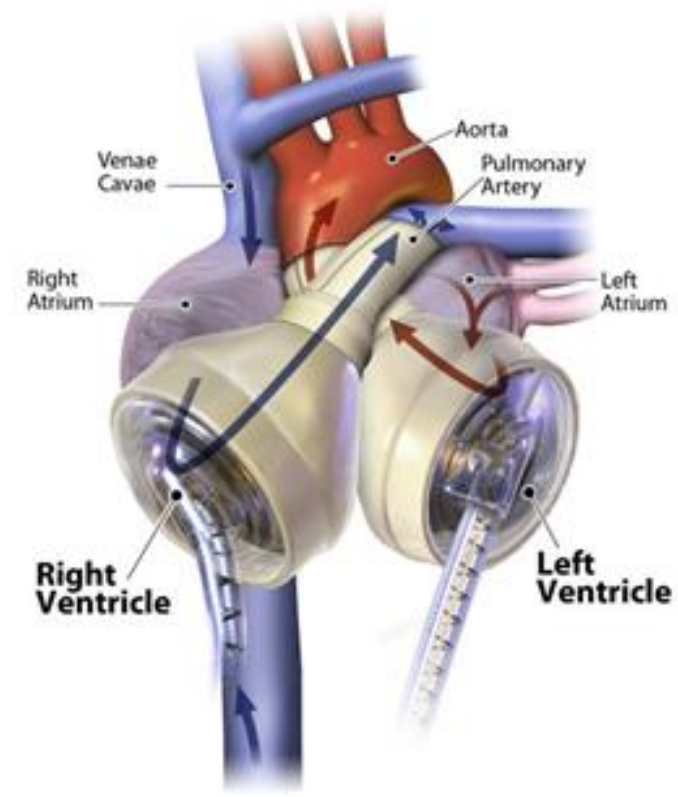

Total Artificial Heart

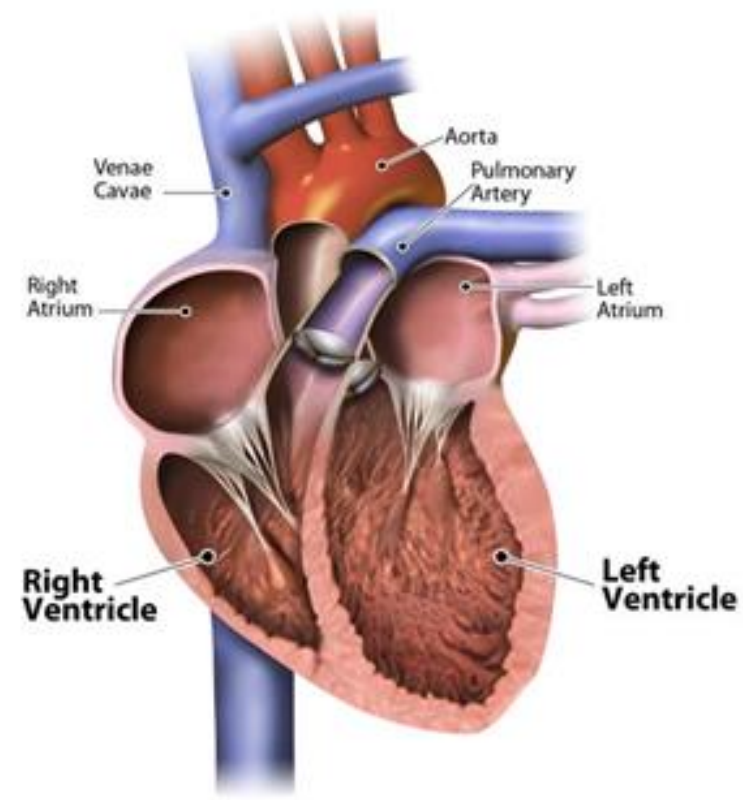

Human Heart

Fig 1: Artificial heart

Artificial Skin:- It is a temporary or permanent replacement of damaged skin. Initially auto skin grafts and all skin grafts were used for the development of artificial skin but later on spray on skin (culturing of healthy skin and keratinocytes and spraying on to small tissues ), temporary skin grafts (culture of unwounded epidermal layer as large sheets), permanent skin grafts (extraction of skin and fibroblasts from the other parts of body and culture on mesh scaffolding), artificial electronic skin (semiconductor nanowires), gelatin containing artificial skin (gelatin isolated from collagen with salt leaching method) composite biocompatible epidermal skin grafts (culture of keratinocytes with silicone membrane i.e. Integra) techniques were developed. Recently nano technology (carbon nanotubes), embryonic stem cells, self-healing polymers etc are being used for the generation of artificial skin. 


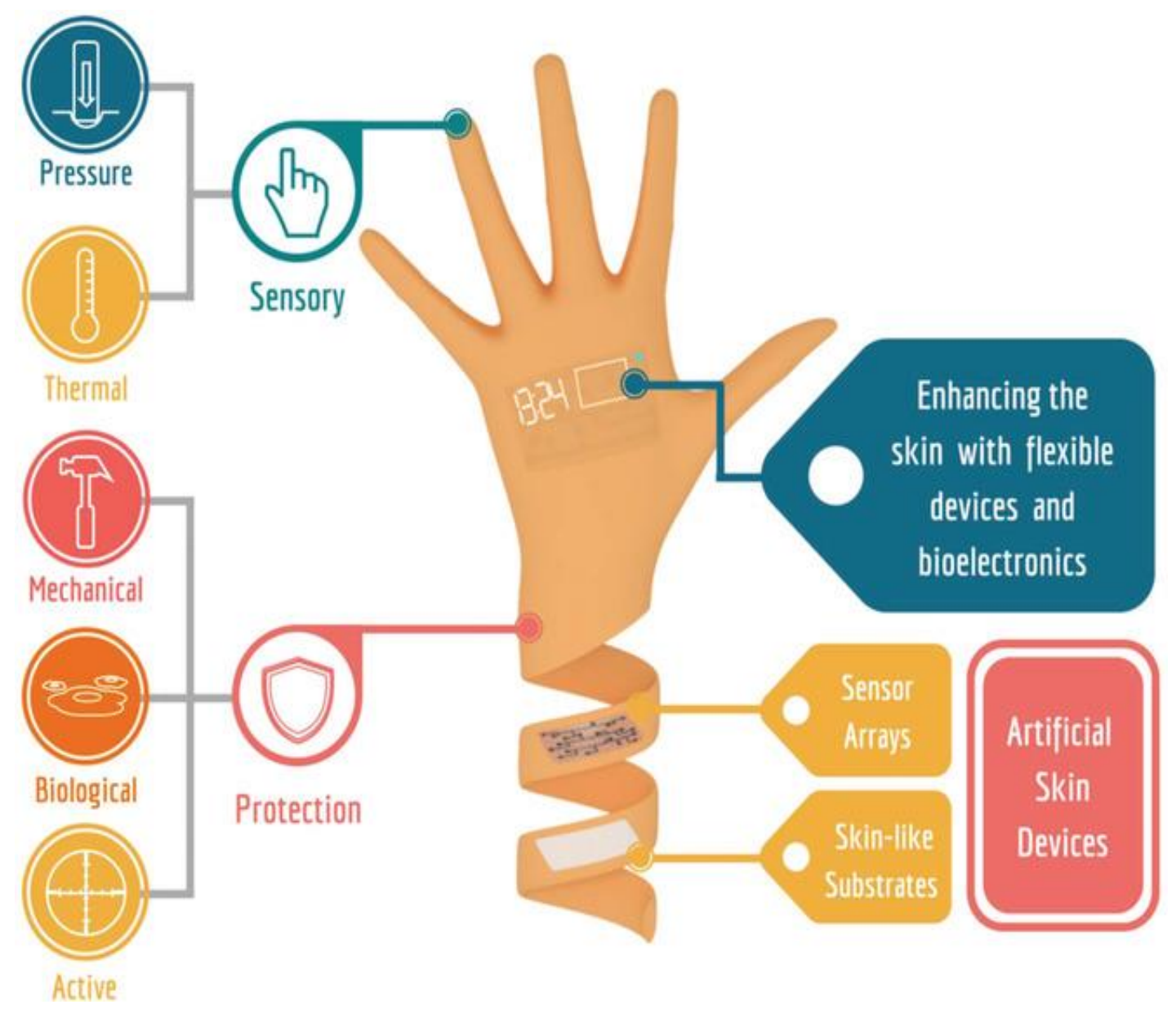

Fig 2: Artificial Skin

Artificial Pancreas:- It is particularly useful for diabetes patients. It automatically controls the blood glucose level and provides substitute endocrine functionality. Generally bio-artificial pancreas consists of a biocompatible sheet of encapsulated beta cells. Gene therapy is also being used for the development of artificial pancreas. An insulin pump under closed loop control is another approach of artificial pancreas.

Artificial Lungs:- It is a device which maintains appropriate blood pressure, decreases injuries of blood cells and minimizes clotting and immunologic response. Extracorporeal membrane oxygenation (ECMO), intravascular oxygenator (IVOX), high-flow cannula in the femoral artery and vein which behaves as pumpless shunt, extracorporeal $\mathrm{CO} 2$ removal etc are some devices which are related to artificial lungs. 


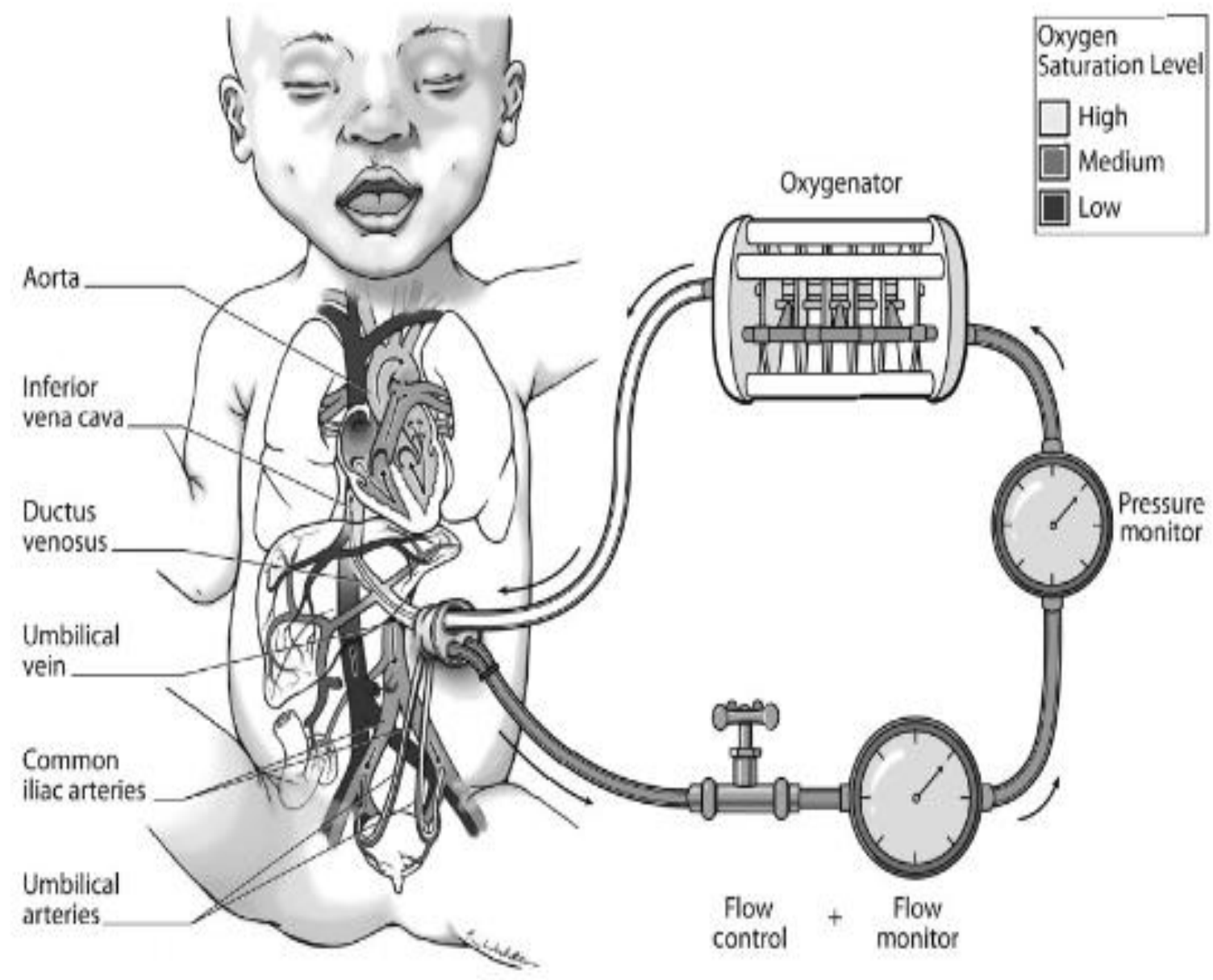

Fig 3: Artificial Lungs

Artificial Urinary bladder:- There are two primary methods for the replacement of urinary bladder. First is the redirection of urine flow and second is in-situ replacement of bladder. Illegal conduit urinary diversion, Indiana pouch reservoir and neobladder to urethra diversion are three prime surgeries for the redirection of urine flow. In these procedures a small part of small and or large intestine is separated from fecal stream and used for the reconstruction of urinary bladder. In alternative approach culture of urothelial and muscle cells is seeded on a biodegradable bladder-shaped scaffold which is made of collagen or a composite of collagen and polyglycolic acid. Similarly other artificial organs like auditory brainstem implant, bionic contact lens, cochlear implant, direct acoustic cochlear implant, retinal implant, visual prosthesis and other assisted devices are developed which possibly help the patients to return in normal life. Although a lot of research is being conducted in this direction but still there is a need of highly advance and specific technologies that could make this field more approachable to normal human being. 

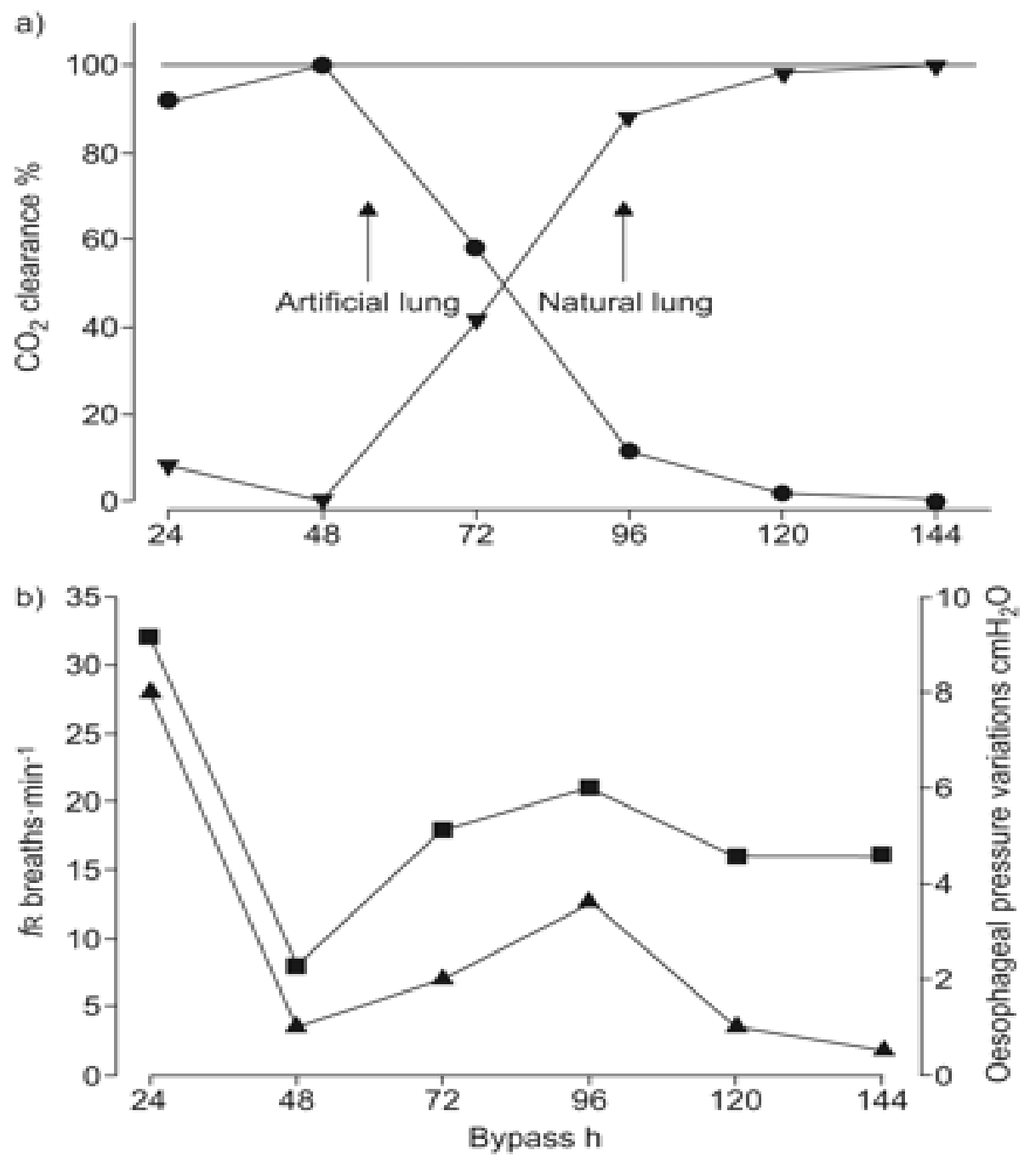

III. CONCLUSION

The synthesis of artificial bio materials is an interdisciplinary field which interfaces biotechnology, material science and nanotechnology. Other processes like biominerlization, synthesis and development of bio-inspired materials and biomimetic systems are patrilineal to the synthetic artificial bio materials. The recent developments of novel bio nano-composites with multi-functionalities lead us to the next generation in this field. Artificial bio materials are significantly important in the development of artificial organs.

\section{REFERENCES}

[1] Gesine Pless, Artificial and Bio-artificial Liver Support, Organogenesis, 3(1), 2007, 20-24. 
[2] Palakkan, A. A., Hay, D. C., PR, A. K., TV, K. and Ross, J. A., Liver tissue engineering and cell sources: issues and challenges, Liver International, 33(5), 2013, 666-76.

[3] Heather Nolan, Dongfang Wang \& Joseph B Zwischenberger, Artificial Lung Basics, Organogenesis, 7(1), 2011, 23-27.

[4] William J. Federspiel \& Robert G. Svitek, Lung, Artificial: Current Research and Future Direction, Encyclopedia of Biomaterials and Biomedical Engineering, 2004, 922-931.

[5] Claudio Cobelli, Eric Renard and Boris Kovatchev, Artificial Pancreas: Past, Present, Future, Diabetes, 60(11), 2011, 2672-2682.

[6] Rayan R. Joshi, Artificial Heart Research: An Historical Perspective, DASH, 2001.

[7] Anthony Atala, Stuart B. Bauer, Shay Soker, James J. Yoo, Alan B. Retik, The Lancet, 367(9518), 2006, 1241-1246.

[8] Rebecca Zumoff, Implantable Artificial Kidney Project Making Progress, Nephrology News \& Issues, Feb 2016.

[9] Catharine Paddock, Implantable Artificial Kidney based on Microchips sees major progress, Feb 2016.

[10] Ross D. Farhadieh, Neil W. Bulstrode \& Sabrina Cugno, Plastic \& Reconstructive Surgery: Approaches \& Techniques, Wiley, 2015. 